craniectomy and biopsy of the lesion. Histopathological examination revealed a lesion composed of confluent granulomata. Smears from the tissue revealed dichotomously branching fungal hyphae, suggestive of Aspergillus spp. [Figure 2]. The fungal culture grew Aspergillus flavus.

The patient was treated with intravenous amphotericin $B$ and oral itraconazole. Computed tomography revealed no regression in lesion size after treatment with $1.5 \mathrm{~g}$ of amphotericin. He thus underwent total excision of the lesion, followed by treatment with $500 \mathrm{mg}$ more of amphotericin B in divided doses. In the postoperative period he developed hydrocephalus and a pseudomeningocele necessitating a ventriculoperitoneal shunt. He required a shunt revision six months later. He was treated with oral itraconazole $200 \mathrm{mg}$ twice a day for one year. One year later, at the time of follow-up, CT scan of the brain showed no recurrence of the mass. However, there was isolated

\title{
An isolated non-dural-based cerebellar aspergilloma in an immunocompetent patient
}

Sir,

Aspergillus infections in the posterior fossa are either extensions of lesions from the mastoid sinuses or part of multiple fungal masses in the brain. ${ }^{[1,2]}$ We report a case of non-dural-based isolated aspergilloma of the cerebellar vermis in an immunocompetent patient. To our knowledge there are only two previous reports of immunocompetent patients with an isolated non-duralbased aspergilloma of the cerebellum. ${ }^{[3,4]}$

A healthy 23-year-old male presented with a onemonth history of holo-cranial headache, vomiting and gait ataxia. Nine months earlier he had developed chronic fever with cough and mild wheezing. He was treated with steroids initially for one month, along with anti-tuberculous therapy for nine months with which his symptoms had rapidly resolved. General and systemic examination, including the ear, nose and paranasal sinsues was unremarkable. Neurological examination revealed bilateral papilloedema and cerebellar ataxia.

Enzyme-linked immunosorbent assay for human immunodeficiency virus (HIV) was negative. Chest radiograph showed resolved opacities in the left lower zone with prominent bronchial markings. Magnetic resonance imaging (MRI) of the brain showed a welldefined mass in the cerebellar vermis, iso/hypointense on T1 weighted images and enhancing with gadolinium injection [Figure 1].

Considering the past history of tuberculosis and the radiological features, a diagnosis of tuberculoma was considered. The patient underwent posterior fossa dilatation of the fourth ventricle [Figure 3] for which he refused therapy.

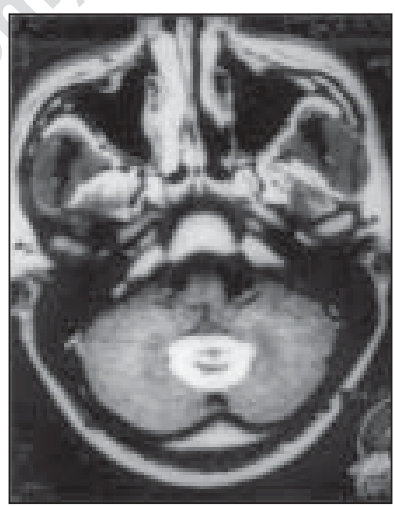

Figure 1: A gadolinium enhanced, T1 weighted axial MR image of the brain, shows a contrast enhancing, discrete lesion in the cerebellar vermis, with minimal reaction in the adjacent brain tissue. Also note absence of any changes in the paranasal and mastoid air-sinuses

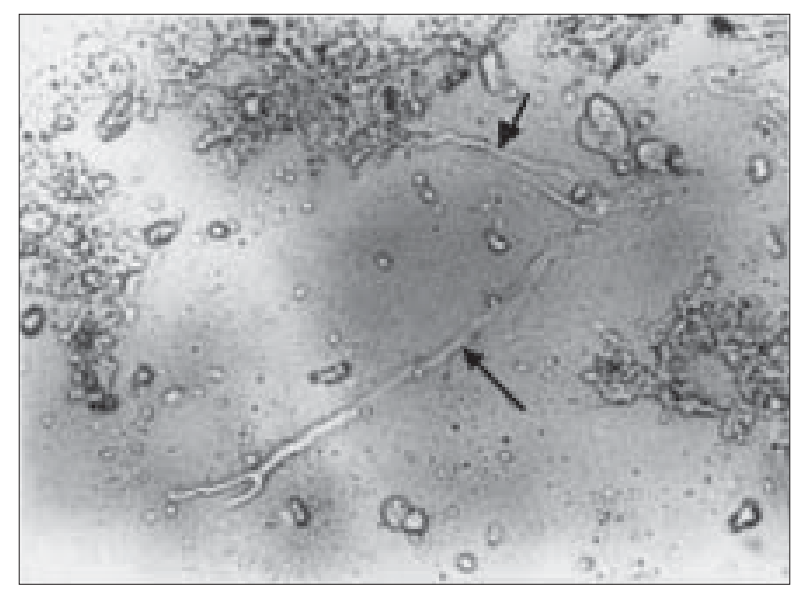

Figure 2: Photomicrograph of the granuloma showing dichotomously branching fungal hyphae suggestive of Aspergillus spp. (arrows) (Unstained KOH preparation, x400) 


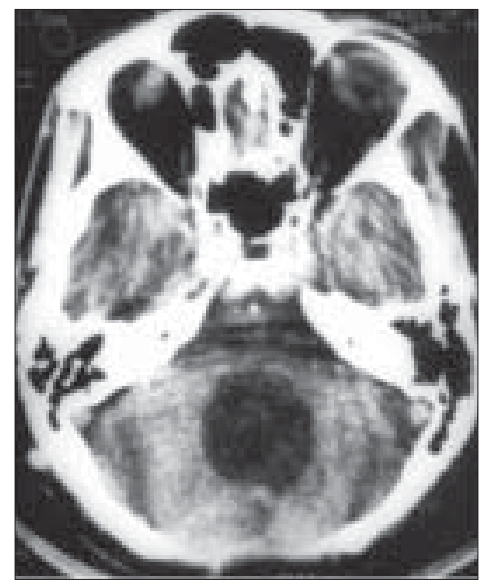

Figure 3: A contrast enhanced computerized tomographic axial image of the brain done at follow-up one year after treatment. Note absence of any residual enhancing lesion in the cerebellar vermis. There is dilatation of the fourth ventricle

Of the two reported immunocompetent patients with an isolated cerebellar aspergilloma, one was an adult ${ }^{[3]}$ and another was an infant (his immune status being debatable). ${ }^{[4]}$ The source of infection in our patient is unclear. Though Aspergillus is known to reside in tuberculous cavities, the chest X-rays did not reveal cavitatory lesions or a fungal ball. The other possibility is that he suffered from a pulmonary aspergillus infection initially which was misdiagnosed as tuberculosis but this is unlikely as he recovered with antituberculous therapy.

Patients with isolated fungal granulomas of the brain may be subjected to prolonged anti-tuberculous therapy empirically, leading to delay in diagnosis and administration of antifungal treatment, resulting in a poor outcome. ${ }^{[1]} \mathrm{A}$ high index of suspicion is required to order fungal culture studies. Total excision of the mass followed by adequate antifungal therapy, as was undertaken in our case, is likely to result in a cure.

\section{Vaijayantee Kulkarni, Vedantam Rajshekhar, Mary S. Mathews*}

Departments of Neurological Sciences and *Microbiology, Christian Medical College Hospital, Vellore, India. E-mail: rajshekhar@cmcvellore.ac.in

\section{References}

1. Sharma BS, Khosla VK, Banerjee AK, Vasishtha RK, Manjunath Prasad KS, Sharma SC, et al. Intracranial fungal granuloma. Surg Neurol 1997;47:489-97.

2. Haran RP, Chandy MJ. Intracranial Aspergillus granuloma. Br J Neurosurg 1993;7:383-8.

3. Salmon MA. Aspergillus of cerebellum. J Roy Soc Med 1983;76:611-3.

4. Mohindra S, Gupta R, Mohindra S, Gupta SK, Vaipehi K. Cerebellar aspergillosis in an infant. Neurosurgery 2006;58:E587. 